\title{
Aspectos éticos de la Declaración de Cartagena
}

\author{
Ethical aspects of the Cartagena Declaration \\ Aspetos éticos da Declaração de Cartagena
}

\author{
Diana Cardenas ${ }^{1}$, Sonia Echeverri² \\ Recibido para publicación: 25 de septiembre de 2019. Aceptado para publicación: 26 de octubre 2019. \\ https://doi.org/10.35454/rncm.v2supl1.032
}

\section{Resumen}

Introducción: el cuidado nutricional fue reconocido como un derecho humano en la Declaración de Cartagena del 3 de mayo de 2019. Este artículo analiza la Declaración de Cartagena desde la perspectiva y fundamentación ética.

Metodología: se hace un análisis desde la ética teniendo en cuenta los principios de la bioética o "principialismo" y los de la Declaración de Bioética y Derechos Humanos de la UNESCO.

Resultados: se reconoce que el derecho al cuidado nutricional implica alimentar a la persona enferma en condiciones que respeten su dignidad, teniendo en cuenta la vulnerabilidad de la persona desnutrida o en riesgo de desnutrición y respetando los principios de la bioética. Por lo tanto, se deben respetar los principios de autonomía, beneficencia, no maleficencia y justicia. Reconocer este derecho y el fundamento ético de la Declaración no implica que se esté aceptando la obligación de alimentar a todos los pacientes en cualquier etapa de la vida y bajo cualquier costo. Por el contrario, reconocer este derecho implica desde el punto de vista ético, que se debe tomar la mejor decisión para el paciente y esto puede incluir la decisión de no alimentar.

Conclusión: la Declaración de Cartagena tiene un componente ético estructural fundamental el cual se basa en los conceptos de dignidad y vulnerabilidad, el respeto a la autonomía, la beneficencia, la no maleficencia y la justicia como condición para el ejercicio de la nutrición clínica. Además, promueve los valores de justicia y equidad en el cuidado nutricional.

Palabras clave: derechos humanos, ética, bioética, malnutrición.

\section{Summary}

Introduction: Nutritional care was recognized as a human right in the Cartagena Declaration on May 3, 2019. This article analyzes the Cartagena Declaration from the ethical approach.

Methodology: The four principle approach to bioethics is used in this paper. Results: it is recognized that the right to nutritional care implies feeding the sick person in conditions that respect their dignity, considering the vulnerability of the malnourished person or at risk of malnutrition and respecting the principles of bioethics. Therefore, the principles of autonomy, beneficence, non-maleficence and justice must be respected. Recognizing this right and the ethical basis of the Declaration does not imply that the obligation to feed all patients at any stage of life and at any cost is being accepted. On the contrary, recognizing this right implies from an ethical point of view that the best decision for the patient must be taken and this may include the decision not to feed.

Conclusion: The Cartagena Declaration has a fundamental structural ethical component which is based on the concepts of dignity and vulnerability, respect for autonomy, beneficence, non-maleficence and justice as a condition for the practice of clinical nutrition. In addition, it promotes the values of justice and equity in nutritional care.

Keywords: Human rights; Ethics; Bioethics; Malnutrition.

\section{Resumo}

Introdução: o cuidado nutricional foi reconhecido como um direito humano na Declaração de Cartagena de 3 de maio de 2019. Este artigo analisa a Declaração de Cartagena sob a perspetiva e o fundamento ético.

Metodologia: é feita uma análise da ética, levando em consideração os princípios da bioética ou "principialismo" e os da Declaração da UNESCO de Bioética e Direitos Humanos.

Resultados: a Declaração de Cartagena tem como fundamento ético os princípios da Declaração da UNESCO de Bioética e Direitos Humanos. Reconhece-se que o direito ao cuidado nutricional implica alimentar a pessoa doente em condições que respeitem sua dignidade, levando em consideração a vulnerabilidade da pessoa desnutrida ou em risco de desnutrição e respeitando os princípios da bioética.

Portanto, os princípios de autonomia, beneficência, não maleficência e justiça devem ser respeitados. $O$ reconhecimento deste direito e da base ética da Declaração não implica que a obrigação de alimentar todos os pacientes em qualquer fase da vida e a qualquer custo seja aceite. Pelo contrário, reconhecer esse direito implica, do ponto de vista ético, que a melhor decisão para o paciente deve ser tomada e isso pode incluir a decisão de não alimentar.

Conclusão: a Declaração de Cartagena possui um componente ético estrutural fundamental, baseado nos conceitos de dignidade e vulnerabilidade, respeito à autonomia, beneficência, não maleficência e justiça como condição para a prática da nutrição clínica. Além disso, promove os valores de justiça e equidade no cuidado nutricional.

Palavras-chave: direitos humanos, ética, bioética, desnutrição. 
Instituto de Investigación en Nutrición, Genética y Metabolismo, Facultad de Medicina, Universidad El Bosque, Bogotá, D.C., Colombia

*Correspondencia: Diana Cardenas

dianacardenasbraz@gmail.com

\section{INTRODUCCIÓN}

El 3 de mayo de 2019 en la ciudad de Cartagena, Colombia, las 16 asociaciones, sociedades y colegios que conforman la Federación Latinoamericana de Terapia Nutricional, Nutrición Clínica y Metabolismo (FELANPE), tras firmar la Declaración de Cartagena, se comprometieron a defender el derecho al cuidado nutricional y a luchar contra la malnutrición. La Declaración de Cartagena busca a través de trece principios proporcionar un marco de referencia para promover el desarrollo del cuidado nutricional en el ámbito clínico que permita que todas las personas enfermas reciban terapia nutricional en condiciones de dignidad. Sirve también, como un instrumento para que las sociedades miembro de la FELANPE y todas las instituciones que trabajan por el cuidado nutricional, promuevan, a través de los gobiernos, la formulación de políticas y legislaciones en el campo de la nutrición clínica. Se trata de un instrumento no vinculante, es decir, que no obliga legalmente, pero que tiene una fuerza moral innegable el cual compromete a las partes a aunar esfuerzos en esta lucha común. Conocer su fundamento ético es clave para la aplicación de los principios de la declaración, el desarrollo del programa de implementación y la formulación de las políticas en nutrición clínica. Este artículo tiene como objetivo realizar un análisis sobre los aspectos éticos y principios que fundamentan la Declaración de Cartagena.

\section{METODOLOGÍA}

La ética es una rama de la filosofía que tiene como objetivo realizar un análisis intelectual de la dimensión humana moral en toda su complejidad ${ }^{(1)}$. La ética tiene que ver con principios que nos permiten tomar decisiones sobre lo que es moralmente correcto o incorrecto. Se refiere a un juicio de comportamientos, buenos o malos. La bioética hace parte de la ética y tiene como objetivo reflexionar y proporcionar respuestas a los problemas y cuestiones o dilemas éticos que presentan los avances de la ciencia y la tecnología incluyendo la

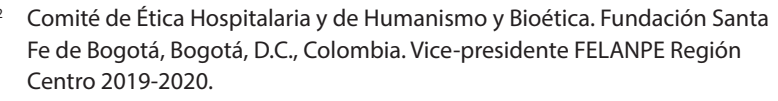

Comité de Ética Hospitalaria y de Humanismo y Bioética. Fundación Santa Fe de Bogotá, Bogotá, D.C., Colombia. Vice-presidente FELANPE Región Centro 2019-2020.

toma de decisiones en el campo de la salud. En este artículo se analiza el fundamento ético de la Declaración de Cartagena teniendo en cuenta los principios de la bioética (autonomía, beneficencia, no maleficencia y justicia) así como los principios establecidos en la Declaración de Bioética y Derechos Humanos de la UNESCO firmada por 191 países en la ciudad de París el 19 de octubre de 2005 (DUBDH) ${ }^{(2)}$.

\section{RESULTADOS - ANÁLISIS}

La nutrición artificial se considera un gran avance en la medicina del siglo XX, ya que gracias a la administración de nutrientes por vía enteral o parenteral hoy podemos alimentar a las personas enfermas que lo requieran, y tener un impacto sobre la malnutrición, el pronóstico de la enfermedad y la calidad de vida. Sin embargo, este avance conlleva a cuestionamientos éticos en situaciones particulares como los cuidados paliativos y el fin de la vida, el paciente con cáncer, el paciente con Alzheimer avanzado, el paciente en cuidados intensivos, etc. ${ }^{(3)}$. Las dificultades en la toma de decisiones y dilemas éticos en este campo surgen principalmente por falta de claridad sobre rol de la nutrición artificial. Se trata de una terapia médica la cual debe tener una indicación, un objetivo terapéutico preciso y debe contar con el consentimiento del paciente, sus familiares o representante legal. Como toda terapia médica, la nutrición artificial tiene indicaciones médicas precisas pero también tener efectos secundarios y complicaciones que pueden llegar a ser más importantes que el beneficio posible y causar daño al paciente. Es por esto que la decisión de retirar o no iniciar la terapia nutricional en situaciones particulares deben tomarse después de un análisis de cada caso y después de una consulta interdisciplinaria con el equipo médico tratante y la familia.

La Declaración de Cartagena al reconocer el derecho al cuidado nutricional como un derecho humano está promoviendo un compromiso y una responsabilidad ética muy importante para todas aquellas sociedades científicas o personas que lo impulsen y lo defienden. 
Es necesario aclarar que esto no quiere decir que se esté defendiendo la obligación de alimentar a todas las personas bajo cualquier circunstancia o en cualquier etapa de la vida, incluso en la fase terminal. Por el contrario, reconocer este derecho implica desde el punto de vista ético, que se debe consensuar con el paciente y familia o cuidadores, la mejor decisión para el paciente y esto incluye la opción de no alimentar. Por lo tanto, se debe promover la alimentación de la persona enferma en condiciones que respeten su dignidad, teniendo en cuenta y respetando los principios de la bioética.

\section{LA DECLARACIÓN UNIVERSAL DE BIOÉTICA $Y$ DERECHOS HUMANOS DE LA UNESCO}

La Declaración de Cartagena se fundamenta en el respeto de los principios establecidos en la $\mathrm{DUBDH}^{(2)}$. Se trata de un referente ético que abarca asuntos relacionados con la medicina, las ciencias de la vida y con la aplicación de la tecnología asociada a los seres humanos, teniendo en cuenta las dimensiones éticas, sociales, legales y ambientales.

La DUBDH tiene como objetivo proveer una estructura universal de principios y procedimientos para orientar a los Estados en la formulación de políticas, leyes y de diversas herramientas aplicadas en el ámbito de la bioética. Se trata de un instrumento que tiene como eje central el respeto a la dignidad humana, a los derechos humanos y se fundamenta en 15 principios (Tabla 1).

La Declaración de Cartagena los reconoce y los aplica en el campo de la nutrición clínica, de tal manera que el Principio 5 "Principios y valores éticos en el cuidado nutricional", afirma:

"Es importante resaltar que la terapia nutricional (nutrición oral, enteral y parenteral) se considera un gran avance científico y tecnológico que ha permitido alimentar a cualquier persona enferma y mejorar los desenlaces clínicos, la calidad de vida e impactar en los costos de la salud. Se reconoce que con estos adelantos surgen cuestiones bioéticas que pueden tener repercusiones en los individuos, las familias y los grupos o comunidades. Dichas cuestiones deberían analizarse en el marco de los principios establecidos en la DUBDH de la UNESCO, en especial los principios universales de igualdad, justicia y equidad, no discriminación y no estigmatización, no maleficencia, autono-
Tabla 1. Principios de la DUBH, UNESCO 2005

\begin{tabular}{|l|}
\hline - Dignidad humana y derechos humanos \\
\hline - Beneficios y efectos nocivos \\
\hline - Autonomía y responsabilidad individual \\
\hline - Consentimiento \\
\hline - Personas carentes de la capacidad de dar su \\
consentimiento \\
\hline - Respeto de la vulnerabilidad humana y la integridad \\
personal \\
\hline - Privacidad y confidencialidad \\
\hline - Igualdad, justicia y equidad \\
\hline - No discriminación y no estigmatización \\
\hline - Respeto de la diversidad cultural y del pluralismo \\
\hline - Solidaridad y cooperación \\
\hline - Responsabilidad social y salud \\
\hline - Aprovechamiento compartido de los beneficios \\
\hline - Protección de las generaciones futuras \\
\hline - Protección del medio ambiente, la biosfera y la \\
biodiversidad. \\
\hline
\end{tabular}

mía, beneficencia y el respeto de la vulnerabilidad humana y la integridad personal."

\section{LA DIGNIDAD HUMANA}

El respeto a la dignidad de la persona es el pilar fundamental en el que están cimentados los valores tanto de la DUBDH como de la Declaración de Cartagena, y es a la vez el hilo conductor de sus enunciados.

El concepto de dignidad humana y el respeto a los derechos humanos están estrechamente relacionados. El enfoque basado en derechos humanos permite identificar las prioridades y objetivos con el fin de luchar contra la desnutrición e implementar un cuidado nutricional óptimo para todos. También permite entender que la dignidad humana es un eje central en el fundamento ético de alimentar a la persona enferma. La noción de dignidad humana desde su dimensión filosófica significa, según Emmanuel Kant, el hecho de que la persona nunca debe ser tratada como un medio, sino 
como un fin en sí mismo. Las personas no tienen un precio, tienen dignidad ${ }^{(4)}$. La dignidad se refiere a una cualidad que estaría vinculada a la esencia misma de cada ser humano, lo que explicaría la razón por la cual esta cualidad debe ser igual para todos y no admitir niveles, grados o excepciones. En este sentido, significa que todos los seres humanos merecen respeto incondicional, independientemente de su edad, salud física o mental, identidad de género u orientación sexual, religión, condición social u origen étnico.

El primer principio de la Declaración de Cartagena está centrado en forma exclusiva en reconocer la importancia de alimentar en condiciones de dignidad a la persona enferma. El respeto de la dignidad humana entendida como el derecho de una persona a ser tratada éticamente, a ser valorada y respetada por sí misma, se logra cuando al alimentar a la persona enferma (a través de la terapia nutricional), se enaltecen su autonomía individual, creencias religiosas y entorno sociocultural. Esto implica reconocer durante las etapas del proceso de cuidado nutricional el valor intrínseco de cada ser humano, así como el respeto a la integridad, la diversidad de valores morales, sociales y culturales.

La ausencia de una atención nutricional óptima puede atentar contra la dignidad humana. Pero también prologar la terapia nutricional más allá de lo médicamente necesario, puede ser considerado como un no respeto de la dignidad humana pues se estaría lesionando la integridad física de la persona. Por lo tanto, se debe tener en cuenta que la terapia nutricional es un tratamiento médico, las personas enfermas tienen el derecho y la autonomía para rechazarlo, y el personal de salud la obligación de aceptar esta decisión y no realizar acciones fútiles.

\section{LA VULNERABILIDAD DEL PACIENTE DESNUTRIDO}

La noción de vulnerabilidad es central en el componente ético de la nutrición clínica. La Declaración de Cartagena afirma:

"Se reconoce que los pacientes en riesgo o en estado de desnutrición son un grupo considerado vulnerable desde el punto de vista ético. La vulnerabilidad es una dimensión ineludible de los seres humanos y la configuración de las relaciones sociales. Considerar la vulnerabilidad del paciente desnutrido implica reconocer que los individuos pueden carecer en algún momento de la capacidad y los medios para alimentarse por sí mismos y, por lo tanto, es preciso que esta necesidad la atiendan profesionales en nutrición clínica. La malnutrición implica deterioro físico, psicológico y social (con el riesgo de perder la vida y la posibilidad de perder la autonomía).”

La palabra vulnerable viene del latín vulnerabilis, formada de vulnus (herida) y el sufijo abilis o able que indica posibilidad, es decir, que puede ser herido. De ahí que la palabra vulnerabilidad en su uso común quiere decir "calidad de poder" ser dañado. Una persona vulnerable es una persona que puede ser herida fácilmente y que no puede defenderse por sí sola en forma expedita. Es de esta manera como en el ámbito del derecho se considera a las personas vulnerables, a las cuales el Estado tiene la obligación de proteger para evitar, por ejemplo, que sean objeto de investigación. En el ámbito clínico, la noción de vulnerabilidad se fundamenta en la noción levinasiana donde se plantea una moral no-intelectualista cuyo origen es la vulnerabilidad y la subjetividad de un individuo, definida por la sensibilidad corporal y no por la conciencia o la razón o la deliberación racional de la libertad $^{(5)}$. De esta forma, según la ética de la vulnerabilidad la cuestión del respeto y el acompañamiento de la persona vulnerable va más allá de la protección de una categoría de individuo y del difícil problema del consentimiento informado ${ }^{(6)}$. En estas condiciones el respeto a la persona no se reduce a la evaluación de su competencia jurídica ni al examen de sus capacidades cognitivas. De igual forma, la respuesta a las necesidades específicas no puede tomar el cariz de una decisión por el otro que sería una destitución de su voluntad. Mucho más que ello, lo que las situaciones de vulnerabilidad hacen resaltar es la necesidad de tener en cuenta el hecho de que la persona necesita del otro, necesita cuidado y estructura médicas, y la realidad de hecho que ella desea ser considerada persona, un ser humano cuya dignidad está intacta a pesar de estar en riesgo de agresión física o cognitiva.

Por lo tanto, el sentido ético más profundo de vulnerabilidad implica el compromiso de la responsabilidad por los demás, la «preocupación por los demás» ${ }^{(6)}$. La vulnerabilidad implica «la responsabilidad por el otro" y eso significa que necesitamos el otro. En el caso de la desnutrición, un paciente desnutrido es doblemente vulnerable porque por un lado, su integridad está comprometida y de otro lado es frágil por su relación de dependencia con el profesional que debe alimen- 
tarlo de manera artificial. La desnutrición implica para los pacientes, del mismo modo que la enfermedad, la pérdida de control del cuerpo, de los procesos vitales necesarios para la salud y la autonomía. En el caso de la desnutrición esto es aún más grave debido a que su diagnóstico es difícil por falta de consenso para definirla y métodos para determinarla; además de la escasa acción de los médicos la cual puede deberse a insensibilidad per se o a desconocimiento del tema de la malnutrición y sus consecuencias. Por lo tanto, es posible considerar que el paciente desnutrido es un paciente doblemente vulnerable. La persona con desnutrición asociada a la enfermedad en situaciones particulares pierde la capacidad de alimentarse. Es entonces dependiente de un cuidador, de sus capacidades técnicas y científicas, pero también de sus cualidades morales para satisfacer este tratamiento médico que es la terapia nutricional.

\section{LOS PRINCIPIOS BIOÉTICOS EN LA DECLARACIÓN DE CARTAGENA}

El principialismo según Tom Beauchamp y James Childress se basa en cuatro principios: el respeto por la autonomía de la persona, el principio de no maleficencia, el principio de beneficencia y el principio de justicia ${ }^{(7)}$. Estos cuatro principios constituyen un marco de referencia común para el análisis de los problemas bioéticos.

\section{AUTONOMÍA}

Los pacientes deben ser tratados como agentes autónomos, es decir, reconocer su capacidad para tomar decisiones independientes y auténticas sobre la forma como quieren ser alimentados, si quieren ser alimentados o no, con base en sus conocimientos, valores y creencias personales. La autonomía no significa que un paciente tenga derecho a obtener cualquier tratamiento que desee o solicite si ese tratamiento en particular no está indicado médicamente. La autonomía solo puede ejercerse después de haber obtenido información completa y apropiada, así como de haberla entendido. La decisión debe tomarse sin ningún tipo de presión o de coerción.

Estos aspectos se manifiestan en los Principios \# 2 y \# 3 de la Declaración de Cartagena. Por un lado, en el Principio 1, se afirma que:

"Se debe tener en cuenta que la terapia nutricional es un tratamiento médico, las personas enfermas tienen el derecho y la autonomía para rechazarlo y el personal de salud, la obligación de aceptar esta decisión."

Esto se complementa con el Principio \# 3 donde se reconoce la importancia del empoderamiento del paciente para asegurar decisiones verdaderamente autónomas por parte del paciente:

"El acto de empoderar a los pacientes y sus familias en la lucha contra la malnutrición implica darles la capacidad para pensar de manera crítica sobre este síndrome y sus respectivas consecuencias negativas, permitiéndoles al mismo tiempo tomar decisiones autónomas e informadas, como exigir atención nutricional y cumplir el tratamiento nutricional sugerido."

\section{BENEFICENCIA}

El principio de beneficencia impone la obligación de actuar en beneficio del paciente. Los cuidadores deben cumplir con las obligaciones y estándares profesionales. Cada decisión debe tomarse en el plano individual. Los profesionales de la salud tienen la obligación de maximizar los beneficios potenciales para sus pacientes y al mismo tiempo minimizar el daño potencial para ellos.

Esto significa que se debe proporcionar una terapia nutricional adecuada en respuesta a una indicación médica y siguiendo el consentimiento del paciente, que la detección del riesgo de desnutrición se debe realizar utilizando una herramienta validada apropiada para todas las personas que entran en contacto con los servicios de salud. La evaluación nutricional debe realizarse en todos los sujetos identificados en riesgo mediante la detección temprana de riesgo nutricional. Se debe realizar la monitorización de la terapia nutricional en todos los pacientes.

El respeto del principio de beneficencia en la Declaración esta implícita en el preámbulo:

"Convencidos de que una adecuada terapia nutricional puede corregir la malnutrición, mejorar el pronóstico de las enfermedades y la calidad de vida, disminuir las comorbilidades, la mortalidad y los costos en salud"

Y en el Principio \# 2 donde se reconoce que:

"El cuidado nutricional forma parte de la atención integral del paciente y, por lo tanto, debe ser un componente inherente a su cuidado." 


\section{NO MALEFICENCIA}

El principio de no maleficencia impone la obligación de no infligir daño a los demás. La terapia de nutrición médica debe minimizar el daño posible. Si el riesgo de administrar terapia nutricional a un paciente específico supera los beneficios potenciales, entonces el profesional de la salud tiene la obligación de no proporcionar (omitir) dicha terapia. Si la terapia nutricional es inútil y solo prolonga el sufrimiento o la aplaza el momento de morir, deberá suspenderse. Además, el respeto de este principio implica evitar el ayuno prolongado e innecesario del paciente hospitalizado. Omitir o retirar la terapia nutricional si se considera inútil (en una situación en la que esta solo prolongaría el sufrimiento, o en la fase terminal de una enfermedad incurable o intratable), situaciones en las que la terapia nutricional no estaría médicamente indicada.

El respeto de este principio se reconoce desde el preámbulo de la Declaración de Cartagena:

"Conocedores de que la terapia nutricional puede tener efectos secundarios y escasa efectividad en algunos pacientes, como aquellos en estado hipercatabólico, o cuando no es administrada de manera oportuna y adecuada"

También se hace énfasis en no realizar acciones fútiles en el Principio \# 1:

"Se debe tener en cuenta que la terapia nutricional es un tratamiento médico, las personas enfermas tienen el derecho y la autonomía para rechazarlo y el personal de salud, la obligación de aceptar esta decisión y no realizar acciones fútiles."

\section{JUSTICIA}

El principio de justicia se refiere a la igualdad de acceso a la atención médica para todos. Los recursos limitados, incluido el tiempo que los médicos y otros profesionales de salud dedican a sus pacientes, deben distribuirse de manera uniforme para lograr un verdadero beneficio para el paciente. Los recursos deben distribuirse de manera justa sin discriminación alguna. Esto quiere decir que todos los paciente deber tener el mejor cuidado nutricional disponible. Esto implica que la terapia nutricional siempre se debe administrar, como cualquier otra terapia, solo cuando exista una indicación médica. Esto está explícito desde el preámbulo de la Declaración de Cartagena:

"Conscientes de la necesidad de buscar, mediante la aplicación de las ciencias básicas, clínicas y de salud pública, soluciones nutricionales cada vez más eficaces"

Y en el Principio \# 6 de la Declaración donde se hace énfasis en el enfoque de la atención en salud basada en el valor:

"Bajo este enfoque, se busca reorientar los servicios de salud para que logren mejorar la satisfacción de las necesidades en salud de las personas, en particular del cuidado nutricional, mientras mantienen una relación óptima con los costos y los resultados.”

El Principio \# 11, reconoce la importancia de los valores de justicia y equidad en el cuidado nutricional.

La Tabla 2 muestra el contenido ético de la Declaración de Cartagena.

Tabla 2. Contenido ético de la Declaración de Cartagena

\begin{tabular}{|l|l|}
\hline \multicolumn{1}{|c|}{ Fundamento ético } & \multicolumn{1}{|c|}{$\begin{array}{c}\text { Declaración de } \\
\text { Cartagena }\end{array}$} \\
\hline $\begin{array}{l}\text { Alimentar a la persona en condiciones de } \\
\text { dignidad }\end{array}$ & - Principio 1 \\
\hline $\begin{array}{l}\text { La vulnerabilidad de la persona en riesgo } \\
\text { o en estado de desnutrición }\end{array}$ & - Principio 5 \\
\hline Respeto del principio de autonomía & - Principio 1 \\
\hline Respeto del principio de beneficencia & - Principio 3 \\
\hline Respeto del principio de no maleficencia & - Principio 2 \\
\hline Respeto del principio de justicia & - Principio 1 \\
\hline
\end{tabular}

\section{CONCLUSIÓN}

La Declaración de Cartagena tiene un componente ético estructural fundamental el cual se basa en los conceptos de dignidad y vulnerabilidad. Los principios que establece esta Declaración reconocen la importancia del principialismo y promueve el respeto a la autonomía, la beneficencia, la no maleficencia y la justicia como condición para el ejercicio de la nutrición clínica. 
Además, promueve los valores de justicia y equidad en el cuidado nutricional.

\section{Financiación}

El presente artículo no tuvo financiación.

\section{Conflicto de intereses}

Los autores declaran no tener conflicto de intereses.

\section{Declaración de autoría}

DC y SE participaron por igual en la concepción y redacción del artículo. Las autoras validaron la versión final.

\section{Referencias bibliográficas}

1. Ricoeur P, Éthique, Dictionnaire d'éthique et de philosophie morale, M Canto-Sperber ed., Paris, PUF, 2004, p. 689.
2. Organización de las Naciones Unidas para la Educación, la Ciencia y la Cultura. Declaración Universal sobre Bioética y Derechos Humanos.[Internet]. Paris: Unesco; 2005 [consultado el 29 ocubre 2015]. Disponible en: http://portal. unesco.org/es/ev.php-URL_ID=31058\&URL_DO=DO TOPIC\&URL_SECTION=201.html.

3. Druml CH, Ballmer P.E, Druml W, Oehmichen F, Shenkin A, Singer P, et al. ESPEN guideline on ethical aspects of artificial nutrition and hydration. Clin Nutr. 2016;35:545-56.

4. Fiat E. Petit traité de dignité. Paris: Ed. Larousse. 2012.p. 234.

5. Levinas E. Autrement qu'être ou au-delà de l'essence, Martinus Nihjoff, The Hague, 1974. [Internet]. [Consultado el 1 de agosto 2019]. Disponible en : https://monoskop.org/ images/8/89/Levinas_Emmanuel_Autrement_qu_etre ou_au_dela_de_1_essence_1974.pdf

6. Pelluchon C. L'autonomie brisée. Bioéthique et philosophie, philosophie. Paris: PUF, 2009. p. 99.

7. Beauchamp TL, Childress JF. Principles of Biomedical Ethics. 7th edition. New York: Oxford University Press, 2013. p. 416. 